\title{
Potenziale erkennen und handeln
}

\author{
Die öffentliche Auftragsvergabe kann sich auch \\ an innovativen Aspekten der Produkte und \\ Dienstleistungen orientieren. Dadurch können \\ innovative Unternehmen gefördert, nachhal- \\ tige Lösungen ausgewählt und die Verwaltung \\ modernisiert werden. \\ Von Matthias Berg und Bianka Blankenberg
}

D er europäische Gesetzgeber hat ein Paket zur Modernisierung des europäischen Vergaberechts geschnürt, das bis April 2016 in nationales Recht umgesetzt werden muss. Der neue Rechtsrahmen soll es den Vergabestellen unter anderem ermöglichen, die öffentliche Auftragsvergabe stärker zur Unterstützung strategischer Ziele zu nutzen - vor allem bezüglich sozialer, ökologischer und innovativer Aspekte.

Dies ist grundsätzlich positiv, da so in jeder Phase des Vergabeverfahrens von der Definition der Leistung über Eignungsund Zuschlagskriterien bis zu den Ausführungsbestimmungen qualitative, innovative, soziale und ökologische Anforderungen gestellt werden können. Die gezielte Nachfrage öffentlicher Auftraggeber nach innovativen Produkten und Lösungen kann so eine Modernisierung der Verwaltung unterstützen, Innovationsentwicklung in der Wirtschaft stimulieren und zu Verbesserungen in den Bereichen Energieeffizienz, Ressourcenschutz, Sicherheit, Gesundheit, Verbraucher- und Arbeitsschutz beitragen.

Innovationsorientierte Beschaffung unterscheidet zwischen dem innovativen Beschaffungswesen und der Beschaffung von Innovationen. Das innovative Beschaffungswesen ist geprägt durch eine optimierte und effizientere Einkaufspraxis sowie durch die Verbesserungen der Beschaffungsprozesse und -organisation im Tagesgeschäft. Unter Beschaffung von Innovationen wird der Einkauf von innovativen Produkten, Technologien und Dienstleistungen verstanden. Sie können Lösungen für neue Bedarfe und Herausforderungen bieten und dabei Produktivität und Effizienz deutlich erhöhen. Der vorliegende Artikel fokussiert auf die Beschaffung von Innovationen.

\section{Impulsgeber für innovative Beschaffung}

Zur Unterstützung der öffentlichen Beschaffung in Deutschland hat das Bundesministerium für Wirtschaft und Energie das Kompetenzzentrum innovative Beschaffung (KOINNO) ins Le- ben gerufen, das vom Bundesverband Materialwirtschaft, Einkauf und Logistik (BME) e. V. aufgebaut wurde und geführt wird [1]. Ziel des KOINNO ist es, die Innovationsorientierung der öffentlichen Beschaffer in Deutschland dauerhaft zu stärken und den Anteil der Beschaffung von Innovationen am Gesamtvolumen des öffentlichen Einkaufs zu erhöhen. Denn zum einen modernisiert der Einkauf von Innovationen die Verwaltung. Zum anderen werden dadurch Innovationen in der Wirtschaft stimuliert, indem sie zu Verbesserungen u. a. in den Bereichen Energieeffizienz, Umweltschutz, Sicherheit, Gesundheit, Verbraucher- oder Arbeitsschutz beitragen.

Wichtige Aktivitäten des KOINNO sind deshalb:

- Aktive Beratung öffentlicher Beschaffungsstellen zu allen Fragen rund um innovative Beschaffung,

I Bereitstellung von Best-Practice-Beispielen, Förderprogrammen und Informationen allgemein,

- Bereitstellung einer Projektdatenbank mit für den öffentlichen Sektor relevanten innovativen Produkten, Technologien und Dienstleistungen,

I Durchführung von Veranstaltungen zur innovativen Beschaffung.

\section{Bedeutung von innovativen Produkten und Dienstleistungen steigt langsam}

Ein Beispiel stellen Beton-Pflastersteine mit fotokatalytischen Eigenschaften dar. Die Stadt Detmold wollte mit dem Neubau des Zentralen Omnibusbahnhofes einen leistungsstarken, komfortablen und barrierefreien Verkehrsknotenpunkt schaffen. Gleichzeitig sollte die Schadstoffbelastung durch die anfallenden Stickoxide deutlich reduziert werden. $\mathrm{Zu}$ diesem Zweck wurden innovative Beton-Pflastersteine mit fotokatalytischen Eigenschaften eingesetzt, die in Verbindung mit UV-Strahlung Stickoxide in unschädliche Salze umwandeln. Von dem Einsatz wird eine Schadstoffreduktion von bis zu 40 Prozent erwartet. Die Innovationskosten des Projektes - die sich aus den Mehrkosten gegenüber der Anschaffung einer herkömmlichen Betonfläche ergeben - liegen, bezogen auf die gesamten Projektkosten, bei 3,3 Prozent. Die Innovation, die das Projekt in die Privatwirtschaft abstrahlte, bestand darin, dass die an der Ausschreibung beteiligten Firmen ihre Kompetenz bei der Herstellung von Betonfahrbahnen, Materialwirtschaft und Verfahrenstechnik steigern konnten.

Ein weiteres Beispiel ist die LED-Beleuchtung in der Asklepios-Stadtklinik Bad Tölz. Die Klinik verfügte in ihren Gebäuden über rund 1.000 Leuchtstoffröhren und 277 Globenleuch- 
ten. Ziel der Klinikleitung war es, diese Leuchtmittel gegen eine effiziente LED-Technologie auszutauschen. Mit Unterstützung von Leuchtmittelherstellern wurden Analysen zum Energieverbrauch erstellt, die ein erhebliches Einsparpotenzial aufzeigten. Die Klinikleitung erstellte auf dieser Grundlage mit einem regionalen Anbieter ein bedarfsgerechtes Beleuchtungskonzept. Im Ergebnis werden Stromkosten von jährlich 21.000 Euro eingespart. Die Amortisationszeit beträgt lediglich 2,23 Jahre.

Diese durch das KOINNO zusammengetragenen Beispiele zeigen, dass öffentliche Hand und Privatwirtschaft Innovationen einkaufen. Bisher ist der Anteil an Innovationen in der öffentlichen Beschaffung gegenüber der gewerblichen Beschaffung aber noch gering. Dies zeigt der Austausch mit Beschaffungsverantwortlichen in unseren Beratungsprojekten und den KOINNO-Veranstaltungen. Nachweisbar ist dies unter anderem in der geringen Anzahl an Ausschreibungen, die Nebenangebote zulassen.

Nebenangebote sind ein Instrument, um einem veralteten Leistungsverzeichnis proaktiv Marktentwicklungen und Innovationen gegenüberzustellen. Sie erfordern jedoch zusätzlichen Aufwand und bergen Risiken bezüglich der Vergleichbarkeit von Angeboten. Voraussetzung für die erforderliche Beschaffung von innovativen Produkten und Dienstleistungen sind daher der Aufbau und die Etablierung innovativer Beschaffungsprozesse. Denn damit werden die Voraussetzungen für den Einsatz innovativer Beschaffungsinstrumente (wie wettbewerblicher Dialog, vorkommerzielle Auftragsvergabe oder Innovationspartnerschaft) geschaffen und die Einkäufer können das Beschaffungswesen innovativ gestalten.

\section{Hemmnisse für die Umsetzung}

In der Privatwirtschaft wurde der Wert einer innovativen, strategischen Beschaffung als Beitrag zum Unternehmenserfolg bereits lange erkannt und vielfach auch umgesetzt. Viele Firmen werben mit ökologischen Beschaffungs- und Herstellungsprozessen sowie der Einhaltung von nachhaltigen Standards beim Einkauf. Noch vor dem Aspekt der Nachhaltigkeit dominiert der Einkauf von Innovationen. Hier hinkt die öffentliche Beschaffung noch weit hinterher. Doch ihre Marktmacht ist mit einem Beschaffungsvolumen von jährlich rund 300 Milliarden Euro enorm. Die Aufwendung von einem Prozent des Beschaffungsvolumens für innovative Produkte und Dienstleistungen dieses Gesamtetats bedeutet einen Innovationsimpuls von drei Milliarden Euro. Ein Vergleich mit den Fördergeldern für Forschung und Innovation in Höhe von 2,1 Milliarden Euro, die Bund und Länder gemeinsam deutschen Unternehmen jedes Jahr bereitstellen, verdeutlicht die potenzielle Hebelwirkung der öffentlichen Hand in diesem Bereich.

Problematisch ist, dass die Einkäufer in den Beschaffungsstellen der öffentlichen Hand kaum auf die neuen Anforderungen vorbereitet sind, strategische und damit innovative Ziele in der Praxis umzusetzen. Ihnen fehlen oft das Hintergrundwissen und auch der Handlungsspielraum, um in diesem Sinne tätig zu werden. Eine vom KOINNO-Team unter öffentlichen Auftraggebern durchgeführte Umfrage hat ergeben, dass sich viele Einkäufer durch den „Paragrafen-Dschungel“ des öffentlichen Vergaberechts gehemmt fühlen: Problematisch sei die Regelflut. Auch das Risiko, sich eventuell in einem Nachprüfungsverfahren verantworten zu müssen, hält vielfach davon ab, Innovationen zu fordern. Viele öffentliche Einkäufer fokussieren deshalb bei der Auftragsvergabe auf den Preis, um auf der vermeintlich sicheren Seite zu sein.

Hauptdefizit ist der enorme Fokus auf die Prozess- und Vergabekonformität in der öffentlichen Beschaffung (REPROCExcellence 2013). Aus unserer Sicht ist dies jedoch kein Vergabeziel, sondern die Prozessbasis. Ziele müssen konkrete Innovations-, Qualitäts- oder Nachhaltigkeitskriterien sein.

Trotz der hohen ökonomischen Relevanz können die großen Erwartungen an eine effiziente öffentliche Beschaffung bisher nicht erfüllt werden. Elemente eines modernen Beschaffungswesens sind vorhanden, allerdings noch nicht in ein übergreifendes strategisches Beschaffungsmanagement eingebunden.

\section{Fazit}

$\mathrm{Zu}$ klären ist, wie öffentliche Auftraggeber die Voraussetzungen dafür schaffen können, strategische und innovative Aspekte bei der Vergabe zu berücksichtigen. Dazu gehört die Förderung der Aus- und Weiterbildung der Einkäufer. Diese müssen außerdem ein entsprechendes Mandat erhalten. Der Einkauf muss frühzeitig in die Strategie der Behörde, für die er beschafft, eingebunden werden, um rechtzeitig innovative und ökologische Aspekte im Vergabeprozess berücksichtigen zu können.

\section{Anmerkung}

[1] Mehr zum Kompetenzzentrum im Internet unter: www.koinno-bmwi.de

\section{Literatur}

REPROC Excellence (2013): REPROC-Excellence Endbericht. Frankfurt, Bundesverband Materialwirtschaft, Einkauf und Logistik.

\section{AUTOREN + KONTAKT}

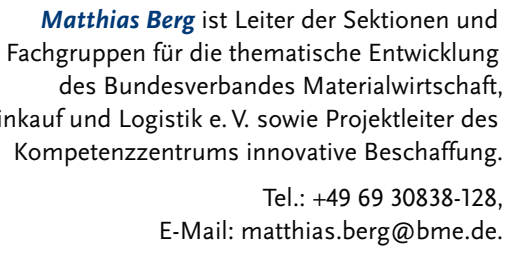

Bianka Blankenberg ist Pressereferentin des Bundesverbands Materialwirtschaft, Einkauf und Logistik e. V.

Tel.: +4969 30838-108,

E-Mail: bianka.blankenberg@bme.de.

Bundesverband Materialwirtschaft, Einkauf und Logistik e. V. (BME), Bolongarostr. 82, 65929 Frankfurt/Main. 